\section{AB1133 HIGH CONSUMPTION OF SEAFOODS OR VEGETABLES NEGATIVELY CORRELATES WITH DISEASE ACTIVITY OF RHEUMATOID ARTHRITIS}

I. Murakami ${ }^{1}$, K. Murakami ${ }^{1}$, M. Hashimoto ${ }^{2}$, M. Torii ${ }^{3}$, K. Ikeda $^{4}$, A. Kuwabara ${ }^{5}$, K. Tanaka ${ }^{6}$, A. Yoshida ${ }^{1}$, T. Usui ${ }^{1}$, N. Kuramoto ${ }^{1}$,

R. Nakashima ${ }^{1}$, Y. Imura ${ }^{1}$, H. Yoshifuji ${ }^{1}$, M. Tanaka ${ }^{2}$, K. Ohmura $^{1}$, T. Mimori ${ }^{1}$. ${ }^{1}$ Department of Rheumatology and Clinical immunology, Graduate School of Medicine; ${ }^{2}$ Department of the Control for Rheumatic Diseases, Graduate School of Medicine; ${ }^{3}$ Department of Human Health Sciences, Graduate School of Medicine; ${ }^{4}$ Department of Diabetes, Endocrinology and Nutrition, Graduate School of Medicine, Kyoto University, Kyoto; ${ }^{5}$ Department of Health and Nutrition, Osaka Shoin Women's University, Osaka; ${ }^{6}$ Department of Food and Nutrition, Kyoto Women's University, Kyoto, Japan

Background: Food intake is one of the important environmental factors of various diseases, and possibly influences the pathogenesis of RA. However, we have little knowledge about the impact of food intake on the pathogenesis of RA. Because each country has its own food culture, the study focused on the dietary habit in Japan is essential in order to clarify the clinical impact of food intake in Japanese RA patients.

Objectives: The aim of this study is to clarify the relationship between the dietary habit of RA patients and their disease status.

Methods: We took the questionnaire survey about dietary habit in 2015, in KURAMA (Kyoto University Rheumatoid Arthritis Management Alliance) cohort as single institute. Disease activity was also examined in this cohort. These data were combined and statistically analyzed.

Results: 563 RA patients were enrolled from KURAMA cohort; female: male $4: 1$, age 63 years old, disease duration 15.5 years, DAS28-ESR 2.8 on average. Multivariate analysis showed that the intake frequency of vegetables had statistically significant negative correlation with DAS28-ESR $(\beta=-0.17, p<0.01)$, SDAI $(\beta=-0.15, p<0.01), \mathrm{HAQ}(\beta=-0.15, p<0.01)$ and MMP $-3(\beta=-0.13, p<0.01)$. The intake frequency of frozen foods had positive correlation with MMP$3(\beta=0.12, p<0.01)$. The intake frequency of juice had positive correlation with DAS28-ESR $(\beta=0.11, p<0.01)$ and SDAI $(\beta=0.11, p<0.01)$. Factor analysis revealed five dietary patterns, which were labeled "seafoods", "meat and fried foods", "vegetables and fruits", "snacks" and "processed foods". The "seafoods" had statistically significant negative correlation with DAS28-ESR ( $\beta=-0.10, p=0.027)$, SDAI $(\beta=-0.11, p=0.015), \mathrm{HAQ}(\beta=-0.11, p=0.015)$ and MMP-3 $(\beta=-0.11$, $p=0.013)$. The "vegetables and fruits" had also statistically significant negative correlation with DAS28-ESR $(\beta=-0.13, p<0.01)$, SDAI $(\beta=-0.14, p=0.015), \mathrm{HAQ}$ $(\beta=-0.17, p<0.001)$ and MMP-3 $(\beta=-0.14, p<0.01)$.

Conclusions: This study implicates that the disease activity of RA may be alleviated by high consumption of vegetables and fruits, or seafoods.

Disclosure of Interest: None declared

DOI: 10.1136/annrheumdis-2017-eular.1631

\section{AB1134 CHARACTERISTICS OF AUTOIMMUNE FEATURED INTERSTITIAL LUNG DISEASE IN KOREAN PATIENTS}

J.-H. Kim, J. Jae Hyun, Y.H. Seo, S.J. Choi, G.G. Song. Inrernal medicine, Korea University Medical center, Seoul, Korea, Republic Of

Background: Interstitial lung disease (ILD) includes a heterogeneous group of disorders that result in diffuse parenchymal lung disease, with overlapping clinical, radiographic, and physiologic manifestations. Several rheumatologic conditions are associated with the development of ILD. There are many patients who are not diagnosed as definite connective tissue disease (CTD). These patients may have an undifferentiated connective tissue disease (UCTD).

Objectives: The aim of this study was to compare the prevalence and characteristics of patients with CTD-ILD, UCTD-ILD and Idiopathic pulmonary fibrosis (IPF) in Korean patients.

Methods: We study compared the prevalence and characteristics of patients with connective tissue disease-associated interstitial lung disease (CTD-ILD), undifferentiated connective tissue disease-associated interstitial lung disease (UCTDILD), or idiopathic pulmonary fibrosis (IPF) between January 2015 and June 2016 in Korea university guro hospital. Clinical characteristics, laboratory tests, and high-resolution CT images were analyzed and compared among three groups.

Results: CTD-ILD was identified in $13.0 \%$, UCTD-ILD in $18.2 \%$, and IPF in $68.7 \%$ among 307 patients. Female and younger age patients were dominant in CTD-ILD group. Pulmonary symptoms were more common in IPF, while extra-pulmonary symptoms were more common in CTD-ILD and UCTD-ILD group. Patients with CTD-ILD had more abnormal antibody tests than those of UCTD-ILD and IPF. Usual interstitial pneumonia pattern was dominant in HRCT images among three groups.

Conclusions: CTD-ILD is not able to be diagnosed accurately in ILD patients. A systematic evaluation of extra-pulmonary symptoms and serologic tests in patients with ILD can identify CTD-ILD, UCTD-ILD, and IPF

References:

[1] Vij R, Noth I, Strek ME. Autoimmune-featured interstitial lung disease: a distinct entity. Chest. 2011;140(5):1292-9.

[2] Castelino FV, Varga J. Interstitial lung disease in connective tissue diseases: evolving concepts of pathogenesis and management. Arthritis Res Ther. 2010;12(4):213.
[3] Kinder BW, Shariat C, Collard HR et al. Undifferentiated connective tissue disease-associated interstitial lung disease: changes in lung function. Lung. 2010;188(2):143-9.

Disclosure of Interest: None declared

DOI: 10.1136/annrheumdis-2017-eular.5647

\section{AB1135 ALLOPURINOL AND THE RISK OF INCIDENT PERIPHERAL ARTERIAL DISEASE IN THE ELDERLY AMERICANS: A U.S. MEDICARE CLAIMS DATA STUDY}

J. Singh, J. Cleveland. University of Alabama at Birmingham, Birmingham, United States

Background: Recently we found that use of allopurinol, the most commonly used urate-lowering therapy (ULT), was associated with a reduction of the risk of myocardial infarction and stroke, acute manifestations of CAD. Given that both PAD and CAD are manifestations of atherosclerosis and a similarity of disease pathophysiology between them, an obvious question was whether allopurinol use would reduce PAD. To our knowledge, no previous studies have examined whether allopurinol use reduces the risk of PAD.

Objectives: To examine whether new allopurinol use is independently associated with a reduction of the risk of incident peripheral arterial disease (PAD) in the U.S. elderly.

Methods: We used the 5\% random Medicare sample from 2006-2012 to examine the association of allopurinol use and its duration with risk/hazard of incident PAD, in a retrospective cohort study. Multivariable Cox regression models adjusted for demographics, comorbidity, cardiac medications and cardiac conditions. Hazard ratios $(\mathrm{HR})$ and $95 \%$ confidence intervals $(\mathrm{Cl})$ were calculated.

Results: We identified 26,985 episodes of incident allopurinol use in 25,282 beneficiaries, of which 3,167 allopurinol use episodes (12\%) ended in incident PAD. In multivariable-adjusted analyses, allopurinol use was associated with HR of $0.88(95 \% \mathrm{Cl}, 0.81,0.95)$ for incident PAD, as was female gender, $0.84(95 \% \mathrm{Cl}$, $0.78,0.90)$. In a separate multivariable-adjusted model, compared no allopurinol use, longer durations of allopurinol use were associated with lower HR of PAD: 181 days to 2 years, $0.88(95 \% \mathrm{Cl}, 0.79,0.97)$ and $>2$ years, $0.75(95 \% \mathrm{Cl}, 0.63$, 0.89). Other factors significantly associated with a higher hazard of PAD were age $75-<85$ and $\geq 85$, female gender, higher Charlson index score, and black race. Sensitivity analyses adjusting for cardiac conditions and medications, confirmed these findings with minimal to no attenuation of hazard ratios.

Conclusions: New allopurinol use was independently associated with a lower risk of PAD in the elderly. Longer allopurinol use durations seemed more protective. Mechanisms of protective effect need to be studied in future studies.

Disclosure of Interest: J. Singh Grant/research support from: Takeda and Savient, Consultant for: Savient, Takeda, Regeneron, Merz, Iroko, Bioiberica, Crealta and Allergan pharmaceuticals, WebMD, UBM LLC and the American College of Rheumatology, J. Cleveland: None declared

DOI: 10.1136/annrheumdis-2017-eular.6848

\section{AB1136 THE AGE OF ONSET OF RHEUMATOID ARTHRITIS CORRELATES WITH AIR POLLUTION AND HEALTH EXPENDITURE: RESULTS FROM MULTINATIONAL DATABASES}

J.D. Castillo-Ortiz ${ }^{1}$, A. Barajas-Ochoa ${ }^{1}$, I. Peláez-Ballestas ${ }^{2}$, A. Ramirez-Gomez ${ }^{1}$, F.J. Aceves-Avila ${ }^{1,3}$, J.J. Castaneda-Sanchez ${ }^{1}$, C. Ramos-Remus ${ }^{4} .{ }^{1}$ Unidad de Investigacion en Enfermedades Cronico-Degenerativas, Guadalajara; ${ }^{2}$ Hospital General de Mexico, Ciudad de Mexico; ${ }^{3}$ Hospital General Regional 46, IMSS; ${ }^{4}$ Universidad Autonoma de Guadalajara, Guadalajara, Mexico

Background: Environmental variables contribute up to half of the variation in the rheumatoid arthritis (RA) susceptibility. We have recently reported that the age of RA onset (RAo) varies across latitudes around the world, starting younger around the Tropic of Cancer (1). Latitude gradients have been used as a surrogate for studying the influence of the environment on the risks of disease in order to generate hypotheses for further investigation.

Objectives: This is an exploratory study to assess whether the age of RAo correlates with tropospheric pollutants (2), electromagnetic fields, Inequalityadjusted Human Development Index (I-HDI) and Health Expenditures as per national census of participating countries.

Methods: The age of RAo was obtained from the GEO-RA group database that involves 2,481 patients from 41 countries. Information of the tropospheric

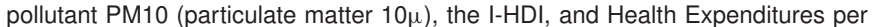
capita was obtained from the World Health Organization's reports. The average of each country's electromagnetic fields (nanotesla, nT) from the past 50 years was calculated using geographic coordinates per country through the magnetic field calculator of The National Centers of Environmental Information. Pearson's correlation and linear regression were used to evaluate the correlation of these environmental variables with the age of RAo by country.

Results: Complete data sets were available in 35 of the 41 countries. Overall, the mean age of RAo was $44 \pm 4.8$ years, the annual average of PM10 of $57.5 \pm 39.3 \mu \mathrm{g} / \mathrm{m}^{3}$, the Health Expenditure per capita of US $\$ 2,212 \pm 2,742$, and the electromagnetic fields of $41,900 \pm 8,720 \mathrm{nT}$. The age of RAo was younger in countries with high PM10 levels $(r=-0.61, p<0.01)$, high inequality (I-HDI, $r=0.59$, 